\title{
Hongrie. Les prises de décision contradictoires dans une nouvelle démocratie
}

Études de cas

Iván Bajomi

\section{CpenEdition}

\section{Journals}

Édition électronique

URL : https://journals.openedition.org/ries/1263

DOI : 10.4000/ries. 1263

ISSN : 2261-4265

\section{Éditeur}

France Education international

Édition imprimée

Date de publication : 1 décembre 2005

Pagination : 51-53

ISSN : 1254-4590

Référence électronique

Iván Bajomi, « Hongrie. Les prises de décision contradictoires dans une nouvelle démocratie », Revue internationale d'éducation de Sèvres [En ligne], 40 | décembre 2005, mis en ligne le 17 novembre 2011, consulté le 08 juillet 2021. URL : http://journals.openedition.org/ries/1263 ; DOI : https://doi.org/ 10.4000/ries. 1263

Ce document a été généré automatiquement le 8 juillet 2021.

(c) Tous droits réservés 


\title{
Hongrie. Les prises de décision contradictoires dans une nouvelle démocratie
}

\author{
Études de cas
}

Iván Bajomi

1 Sous le régime communiste, la Hongrie était dotée d'un système scolaire fortement centralisé. Depuis le changement de régime, le pays fait partie des systèmes éducatifs les plus décentralisés d'Europe. Une grande partie des questions concernant la scolarisation fait l'objet de décisions locales. Cependant, malgré cette forte décentralisation, maintes mesures prises au niveau national influencent toujours les processus de scolarisation. Ainsi, c'est à ce niveau que l'on détermine les mécanismes de financement des écoles; de même, c'est au niveau national que sont élaborés les appels d'offre dont dépendent les acteurs locaux du système d'éducation. Il faut noter que, parallèlement à l'adhésion de la Hongrie à l'Union européenne, l'importance de ces appels n'a cessé de croître puisque les différents programmes éducatifs et les fonds structurels augmentent considérablement le volume des subventions auxquels peuvent désormais prétendre les chefs d'établissement ou autres responsables dont l'action est par ailleurs fortement conditionnée par les restrictions permanentes.

\section{La valse des réformes}

2 Tout comme dans les autres pays du bloc communiste, le changement de régime s'est produit en Hongrie de façon tout à fait inattendue. Il a eu pour résultat l'adoption de maintes décisions sans que des études préalables approfondies aient été menées et sans qu'il existe un large consensus autour des réformes à entreprendre.

Cette nouvelle situation a été propice à de forts mouvements de balancier. Depuis l'instauration du pluralisme politique, chaque élection législative a abouti à un changement de coalition et a amené au pouvoir des partis porteurs de conceptions diamétralement opposées en matière d'éducation. Les mesures mises en œuvre avaient 
de fortes chances d'être remises en cause à la suite de l'alternance politique. Des mouvements de balancier importants ont affecté par exemple les instances de régulation. Ainsi, malgré les protestations des tenants de l'autonomie pédagogique, un gouvernement de droite a créé, en 1993, des centres déconcentrés similaires aux académies françaises. Mais un an plus tard, une nouvelle coalition de gauche les a démantelés; avec le retour de la droite au gouvernement, de nouveaux centres déconcentrés, dont la principale mission consiste à procéder à des évaluations, ont été créés au niveau régional. La réglementation des contenus scolaires a aussi connu des zigzags similaires ${ }^{11}$. Durant les années 1990 , on a pu constater une succession infinie de nouvelles variantes du programme national de base dont les premières versions étaient inspirées par le core curriculum anglais. Ces mouvements de balancier persistent de nos jours. En effet, à la suite d'une nouvelle victoire de l'alliance des socialistes et des libéraux en 2002, les écoles ne sont plus obligées de respecter les "programmescadres » relativement contraignants qui avaient été introduits en 1999, tandis que ce même gouvernement a imposé en 2003 une nouvelle version révisée de fond en comble du programme national de base qui est désormais axé sur le développement des compétences et ne définit des contenus que de façon tout à fait indirecte. Au sujet de ce nouveau tournant que les responsables de la politique éducative légitiment par les mauvais scores obtenus par la Hongrie dans le domaine des compétences lors de l'enquête PISA-2000, de très fortes critiques ont été formulées par les associations d'enseignants, mais le processus de réforme n'a pas été abandonné.

\section{Les carences de la concertation}

Dans cette nouvelle démocratie pluraliste, les mouvements de balanciers observés pourraient en théorie être modérés voire entravés par des actions de mobilisation politique. Il n'en est rien en raison de la faiblesse du monde associatif et des syndicats enseignants. (Il est à noter que malgré le faible pouvoir d'achat des enseignants, aucune grève de grande envergure n'a eu lieu en Hongrie depuis le changement de régime.)

aussi pu s'attendre à ce que les instances nationales de concertation mises en place au début des années 1990 incitent les responsables de la politique éducative à plus de modération. Cependant, sur la base d'une étude que je mène sur ces instances consultatives nationales, je crois pouvoir affirmer que ces organes souffrent de nombreuses carences liées à leur genèse et à leur mode de fonctionnement.

D'une part, on peut déplorer le fort éparpillement des conseils éducatifs. En effet, outre un forum tripartite rassemblant trois types d'institutions relativement puissants (ministères, syndicats enseignants, responsables des pouvoirs locaux), existent deux conseils plus larges dont l'un rassemble trois genres d'acteurs (associations professionnelles du champ pédagogique, instituts de formation des enseignants et délégués de la recherche en éducation), tandis que l'autre conseil rassemble les représentants de sept types d'institutions ou d'acteurs (ministères, syndicats, pouvoirs locaux, parents d'élèves, élèves, Églises et minorités nationales, etc.). En outre, les élèves et les minorités nationales disposent chacun d'un petit conseil. Il faut aussi signaler un grand absent : les partis, qui ont un rôle déterminant dans l'élaboration de la politique éducative mais ne sont nullement représentés dans les conseils mentionnés. 
7 Nul ne s'étonnera que, notamment en raison de cette multiplicité de conseils, le fonctionnement des ces instances reste peu connu des enseignants et d'un large public. Ainsi, le principal hebdomadaire pédagogique du pays, qui dépend directement $d u$ ministère de l'Éducation, omet très souvent de publier les informations sur le fonctionnement du conseil formé de sept composantes. Il va de soi que la séparation des processus de consultation en plusieurs conseils distincts ne favorise guère la formation d'un consensus entre les représentants du monde de la recherche et les représentants des différents groupes d'intérêt. Si ces conseils n'exercent qu'une faible influence sur les grandes orientations de la politique éducative, cela vient aussi de la faiblesse des moyens disponibles et de leur mode de fonctionnement (souvent, ces instances ne sont consultées que juste avant l'adoption de telle ou telle mesure et les textes soumis à discussion ne présentent pas les alternatives possibles; contrairement au ministère, ces conseils ne sont pas en mesure de commander des recherches, etc.). Somme toute, ces instances contribuent surtout à améliorer les décisions sur le plan technique, tandis qu'elles sont incapables d'inscrire à l'ordre du jour de la politique éducative nationale des priorités différentes de celles qui sont retenues par les représentants des partis.

\section{NOTES}

1. Voir Bajomi I., « Un jeu de balancier : la définition réglementaire des contenus d'enseignement dans la Hongrie post-communiste", Revue Internationale d'Éducation de Sèvres, $\mathrm{n}^{\circ} 32,2003$, pp. 42-50.

\section{INDEX}

Index géographique : Hongrie

\section{AUTEUR}

\section{IVÁN BAJOMI}

Professeur, université Eötvos Lorand, Budapest. 